\title{
Numerical Analysis of Drug Diffusion in Human Dermal Region with Linear Shape Function
}

\author{
Vineeta Gupta ${ }^{1}$, S.S. Pagey ${ }^{1}$,V.P.Saxena ${ }^{2}$ \\ ${ }^{1}$ Institute for Excellence in Higher Education, Bhopal, 462042 \\ ${ }^{2}$ Sagar Institute of Research and Technology-Excellence,Bhopal,462041
}

\begin{abstract}
The present model discusses the drug distribution in the layers of human dermal parts. We have considered tissue absorption rate of drug is decreasing function of drug concentration, also considered linear shape functions for approximating element wise approximate solution by Finite Element Method (FEM).In this method each region is referred to as an element and the process of subdividing a domain into a finite number of elements is referred to as discretization. Elements are connected at specific points, called nodes, and the assembly process requires that the solution be continuous along common boundaries of adjacent elements. MATLAB Software used for numerical calculation.
\end{abstract}

Keywords: Transdermal Drug Delivery System, Diffusion Equation, Finite Element Method

\section{Introduction}

Skin is the soft outer covering and largest organ in a human body with multiple layers .It's outer most layer is called epidermis. The epidermis can be subdivided into five strata which are stratum corneum, stratum lucidum, stratum granulosum, stratumspinosum and stratum germinatirum. The top most layer of strata is stratum corneum consisting of approximately 15-350 layers of dead cells. This layer act as a physical barrier to those substances that come in contact with the skin. The different type of cells which make up the epidermis are Merkev cells, Keratinocytes with melanocytes and Langerhans cells. Cells are formed through mitosis at the basale layer. By cell division process, cells move up the epidermis .Changing shape and composition as cells die due to isolation from their blood source. The cytoplasm is released and the protein keratin is inserted. They eventually reach the stratum corneum and slough off (desquamation). This process is called keratinization. This keratinized layer of skin is responsible for keeping out water and other harmful chemicals in the body and making skin a natural barrier to infection. The epidermis helps the skin to regulate body temperature.

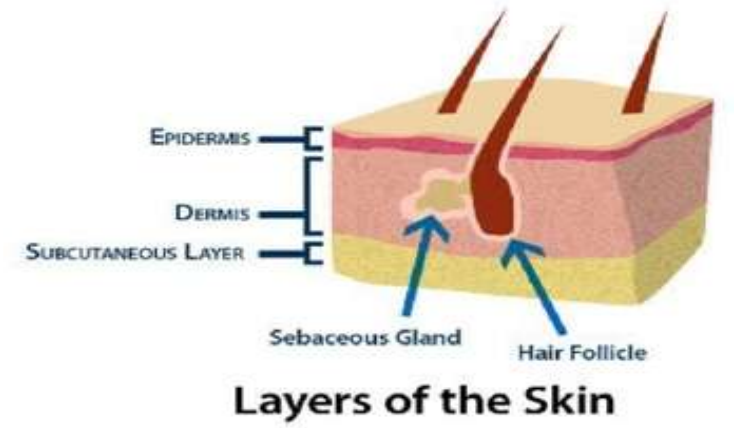

Skin has a layer beneath the epidermis is dermis. The dermis is tightly connected to the epidermis by a membrane. It contains the hair follicles, sweet glands, sebaceous glands, blood vessels. The blood vessels in the dermis provide nourishment and waste removal from its own cells as well as from the stratum basale of the epidermis.

Hypodermis (subcutaneous tissues ) is not a part of the skin and it lies below the dermis .Its purpose is to attach the skin to underlying bone and muscle as well as supplying it with blood vessels and nerves. It consists of loose connective tissue and elastin. Its thickness varies in different regions of the body. It helps to insulate the body against changes in the outside temperature.

Transdermal therapeutic systems are defined as self contained discrete dosage forms when applied to the intact skin. Transdermal Drug Delivery System (TDDS) has been in existence since last thirty years. Those days, the most common applied system was topically applied creams and ointments for dermatological disorders. The occurrence of systemic side effects with some of these formulations is indicative of absorption through the skin. A number of drug have been applied to the skin for systemic treatment .In a broad sense, transdermal therapeutic systems have been designed to provide controlled continuous delivery of drugs via skin to systemic circulation .[1] 
To the successful development of transdermal therapeutic systems, we must have the knowledge of skin permeation kinetics .Following steps are involved for transdermal permeation of a drug from patch to the skin:

(a) Diffusion of drug from drug reservoir to the rate controlling membrane.

(b) Diffusion of drug from rate limiting membrane to stratum corneum.

(c) Sorption by stratum corneum.

(d) Penetration of drug through viable epidermis.

(e) Uptake of drug by the capillary network in the dermal papillary layer. [1]

Basic components of transdermal drug delivery systems are:

(a)Polymer matrix/drug reservoir - these are backbone of TDDS, which control the release of drug from the device.

(b)Drug - Transdermal route is an extremely attractive option for the drugs with appropriate pharmacology and physical chemistry. Transdermal patches offer much to drugs which undergo extensive first pass metabolism, drugs with narrow therapeutic window or drugs with short half life which causes non compliance due to frequent dosing.

(c) Permeation enhancer - These are the chemical compound that increase permeability of stratum corneum.

(d) Other recipients i.e. pressure sensitive adhesive, backing laminate, solvents etc.[1]

A basic knowledge of transfer process from the transdermal patches and into and through the skin is important, figure 1 represents the idealized scheme of these consecutive steps.

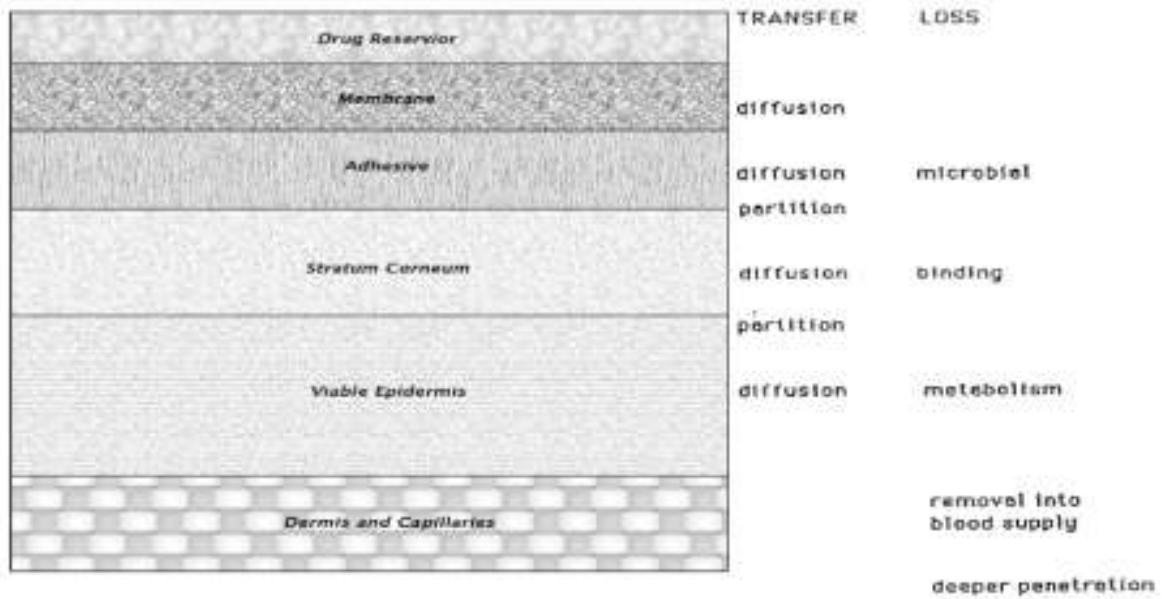

Figure 1 [Transfer and loss processes of drug into the skin by transdermal drug delivery system][6]

As can be seen in figure 1, the predominant events involve partition and diffusion. The drug will partition from reservoir into polymer matrix that comprises the rate-limiting membrane. Once in the membrane, diffusion will occur down a concentration gradient at a rate which will be controlled by the diffusion coefficient of drug in the polymer. Drug has diffused through the rate controlling membrane, it will partition into the adhesive layer. Transport through this region will also be related to molecular size, but will be considerably faster thanthat through the membrane. Drug contained in this area will be released relatively rapidly to the skin. Typically, the release profile will follow first-order kinetics. [6]

The exact amount of drug required is dependent on physicochemical and biological properties of the compound. If these are known, educated estimate will be made. Without a precise knowledge of concentration and degree of storability of drugs, we cannot make effective transdermal patches. For the knowledge of drug concentration into the human dermal part we established here a mathematical model. This model is depending on physicokinetic properties of drug.[6]

\section{Formulation of periodic drug distribution mathematical model}

In 1855, Fick recognized that mathematical equation of heat conduction developed by Fourier in 1822 could be applied to mass transfer. Fick's find that fluxis proportional toconcentration gradient $\frac{\partial C}{\partial x}$

$$
\text { Hence } \quad J=-D \frac{\partial C}{\partial x} \text {. }
$$


Where $D$ is diffusivity, $C$ is concentration and $x$ is distance of movement perpendicular tothe surface of the barrier. Equation (2.1) is known as Fick's first law.

Fick's second law[13] derived as, the concentration in a particular volume element changes only as a result of net flow of diffusing molecules into or out of the region i.e.

$$
\frac{\partial C}{\partial t}=D \frac{\partial^{2} C}{\partial x^{2}}
$$

This equation represents diffusion only in $x$ direction.

Extended form of Equation (2.2) was employed by Chao. et. al (1973)[2] and Chao and Yang(1975)[3] to study heat diffusion in dermal region .Similar equation has been widely used by Saxena and his coworkers $(1981,1983,1987,2009)[14,15,16]$. In most of the cases finite element method has been employed by Saxena. Recently Saxena and Sharma (2011) [17] used this approach to solve One-dimensional drug distribution problem in human dermal region. We use this approach in present model and consider here when drug is diffused in dermal layers, drug mainly absorbed by tissues and blood but tissue absorption rate of drug will be decreasing function of drug concentration and drug absorbsion by other factors almost negligible.

Accordingly, the transport of drugs in one dimension in the human skin is governed by following differential equation

$$
\frac{\partial}{\partial x}\left(D \frac{\partial C}{\partial x}\right)-A(C)-B=\frac{\partial C}{\partial t}
$$

Where

$A(C)=$ Tissue absorption rate of drug depend on drug concentration (C)

$B=$ Drug intake rate by blood

$x=$ Depth below the skin surface

The values of the parameters depend on time $(\mathrm{t})$ and depth $(x)$.

The drug distribution has mild barrier effect at the interfaces and flux is generallycontinuous, hence

$$
C^{(i)}=\rho_{i} C^{(i+1)} \text {, at } x=a_{i}(i=1,2)
$$

Where $\rho_{i}^{\prime} s(i=1,2)$ are theskin partitioncoefficients for the drug at the respectiveInterfaces.Formostof the drug cases, $\rho_{i} \approx 1$.

Finite element method [9] is used to numerically solve the differential equation (2.3).We discretized human dermal part into three layers and consider the concentration of drugvaries linearly in each layer and defined as a function of one space variable $\mathrm{x}$. Therefore layer wise concentration $\mathrm{C}^{(\mathrm{i})}(\mathrm{i}=1,2,3)$ is approximated by a linear polynomial with respect to $\mathrm{x}$ as

$$
\begin{aligned}
& \qquad c^{(i)}=u_{i}+v_{i} x \\
& \text { where } a_{i-1} \leq x \leq a_{i},(i=1,2,3) \\
& \text { volues of } u_{i} \text { and } v_{i} \text { are obtained as } \\
& \qquad u_{1}=\frac{a_{1} c_{0}-a_{0 c_{1}}}{a_{1}-a_{0}} \quad v_{1}=\frac{c_{1}-c_{0}}{a_{1}-a_{0}} \\
& \qquad u_{i}=\frac{c_{i-1} a_{i}-c_{i a_{i-1}}}{a_{i}-a_{i-1}}, \quad v_{i}=\frac{c_{i}-c_{i-1} / \rho_{i}}{a_{i}-a_{i-1}},(i=2,3)
\end{aligned}
$$

Since drug is targeted to subcutaneous region only and it has negligible concentration beyond the subcutaneous region therefore at the innermost boundary the concentration is

$\mathrm{C}_{3}=0$

Since epidermis contains dead cells and no blood vessels, therefore $\mathrm{T}_{\mathrm{i}}(\mathrm{C})$ and $\mathrm{B}_{\mathrm{i}}$ are the values of absorption coefficient in the respective layers and non-zero except at the upper most layer.

Once a tissue absorb drug in some period of time then for next period tissue absorb comparatively less drug from the first period so, we can say that tissue absorption rate will be decreasing function of drug concentration. In general we take 


$$
A_{i}\left(C^{(i)}\right)=e^{-k_{i} C^{(i)}},(i=1,2,3)
$$

Wherek ${ }_{\mathrm{i}}$ 's $(<<1)$ are an individualist parameters in each layer which may be different for different subjects. Thus for marginally small concentrations we can write

$$
A_{i}(C) \approx 1-k_{i} C^{(i)},(i=1,2,3)
$$

As we consider shape function in One-dimensional linear form in which the material properties, initial conditions and boundary condition vary, ignore the presence of metabolic reactions in the skin because the transdermal drug delivery is free from the first pass metabolism effect.

\section{Steady Sate Case with Constant Drug Application}

For steady state case the transport of drug in the skin is governed by the following differential equation,

$$
\frac{\partial}{\partial x}\left(D \frac{\partial C}{\partial x}\right)-T(C)-B=0
$$

The drug concentration at the outer surface is denoted $\mathrm{byC}_{0}$, which is generally known.

We use Euler-Lagrange's equation (Myers, 1971) [10]

$$
\frac{\partial}{\partial x}\left(\frac{\partial F}{\partial C^{\prime}}\right)-\frac{\partial F}{\partial C}=0
$$

Where $C^{\prime}=\frac{\partial C}{\partial x}$

To convert equation (3.1) in the variational form given by

$$
I=\int_{0}^{L}\left[\frac{D}{2}\left(\frac{d C}{d x}\right)^{2}+(1+B) C-k \frac{C^{2}}{2}\right] d x
$$

Here $L$ is total thickness of the skin . For optimum value of $l, L$ is divided into three natural

layers of thickness $a_{1}-a_{0}, a_{2}-a_{1}, a_{3}-a_{2}$

The parameters $D, B, k$ are taken constants in each layer. Thus, variational form of above equation can be written as

$$
I_{i}=\int_{a_{i-1}}^{a_{i}}\left[\frac{D_{i}}{2}\left(\frac{d C^{(i)}}{d x}\right)^{2}+\left(1+B_{i}\right) C^{(i)}-k_{i} \frac{\left(C^{(i)}\right)^{2}}{2}\right] d x
$$

The integrals are evaluated for each sub region and are given below.

$$
\begin{aligned}
& I_{1}=\frac{D_{1}}{2}\left(a_{1}-a_{2}\right) v_{1}^{2}+E_{1}\left(a_{1}-a_{0}\right) u_{1}+\frac{E_{1}}{2}\left(a_{1}^{2}-a_{0}^{2}\right) v_{1} \\
& I_{t}=\frac{D_{i}}{2}\left(a_{i}-a_{i-1}\right) v_{i}^{2}+\left(\frac{1}{2}+\frac{A_{i}}{2}+B_{i}\right)\left\{u_{i}\left(a_{i}-a_{i-1}\right)+\frac{v_{i}}{2}\left(a_{i}^{2}-a_{i-1}^{7}\right)\right\} \ldots \ldots . . . . .(3.5) \\
& \text { Where }(i-2.3) \\
& \text { These Integrals } I_{i}(i=1.2 .3) \text { are assembled to obtain } \\
& \qquad I=\sum_{i=1}^{x} l_{i}
\end{aligned}
$$

Now $\mathrm{I}$ is optimized with respect to each $C_{i}(i=1.2)$ give the following system of differential equations.

$$
\begin{aligned}
& E_{6} C_{1}-E_{4} C_{2}=E_{7} \\
& E_{8} C_{1}-E_{4} C_{2}=E_{9}
\end{aligned}
$$

Where $E_{4}, E_{6}, E_{7}, E_{\mathrm{B}}, E_{9}$ are the constant coefficients listed in the Appendix $A$ and different values of $T_{i}(C)$ and $B_{i},(i=2,3)$ are listed in table 1 and table 2 .

\section{Numerical examples and discussion}

Since last thirty years transdermal drug delivery system have been used as safe and effective drug delivery devices .This study provide a better understanding of the drug delivery mechanisms and in depth analysis of the effect of some physicochemical parameters on concentration profile . The thickness of in-vivo tissues layers can be assigned any values depending upon the structure of skin .Accordingly we take 
$a_{0}=0.00 \mathrm{~mm}, a_{1}=0.01 \mathrm{~mm}, a_{2}=0.03 \mathrm{~mm}, a_{3}=0.07 \mathrm{~mm}$

On solving (3.8) and (3.9), we get the nodal concentration $c_{i}(i=1,2)$ and substituting the values of $C_{f}$ in (2.9) and determine the values of the field concentration $C^{(i)}(i=1,2,3)$

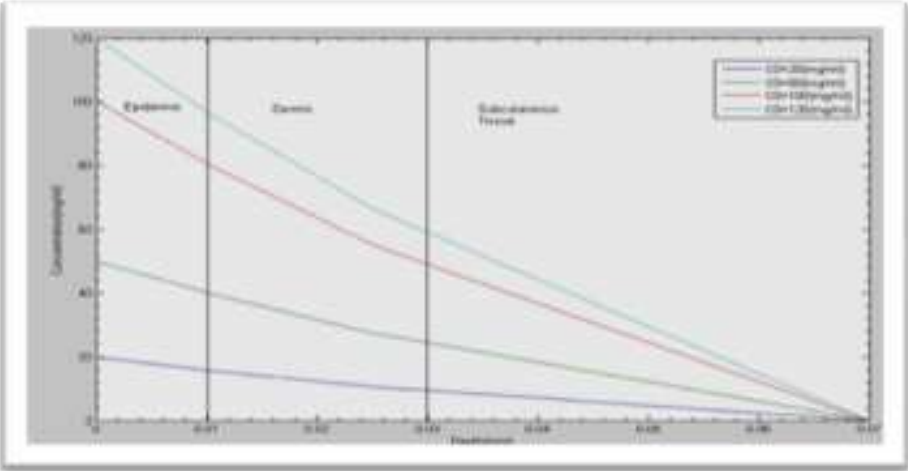

Figure [2]

Steady state drug concentration distribution in skin layer. The result for the linear diffusion with $\mathrm{T}_{1}(\mathrm{C})=0$ $\mathrm{gm} / \mathrm{mm}^{3} \mathrm{sec}, \quad T_{2}(\mathrm{C})=7 * 10^{-3} \mathrm{gm} / \mathrm{mm}^{3} \mathrm{sec}, \mathrm{T}_{3}(\mathrm{C})=8 * 10^{-3} \mathrm{gm} / \mathrm{mm}^{3} \mathrm{sec}, \quad B_{1}=0 \quad \mathrm{gm} / \mathrm{mm}^{3} \mathrm{sec}, B_{2}=4 * 10^{-2} \mathrm{gm} / \mathrm{mm}^{3} \mathrm{sec}$, $\mathrm{B}_{3}=7 * 10^{-2} \mathrm{gm} / \mathrm{mm}^{3} \mathrm{sec}, \mathrm{D}_{1}=5.83 * 10^{-4} \mathrm{~mm}^{2} / \mathrm{sec}, \mathrm{D}_{2}=7.16^{*} 10^{-4} \mathrm{~mm}^{2} / \mathrm{sec}, \mathrm{D}_{3}=9.04 * 10^{-4} \mathrm{~mm}^{2} / \mathrm{sec}$.

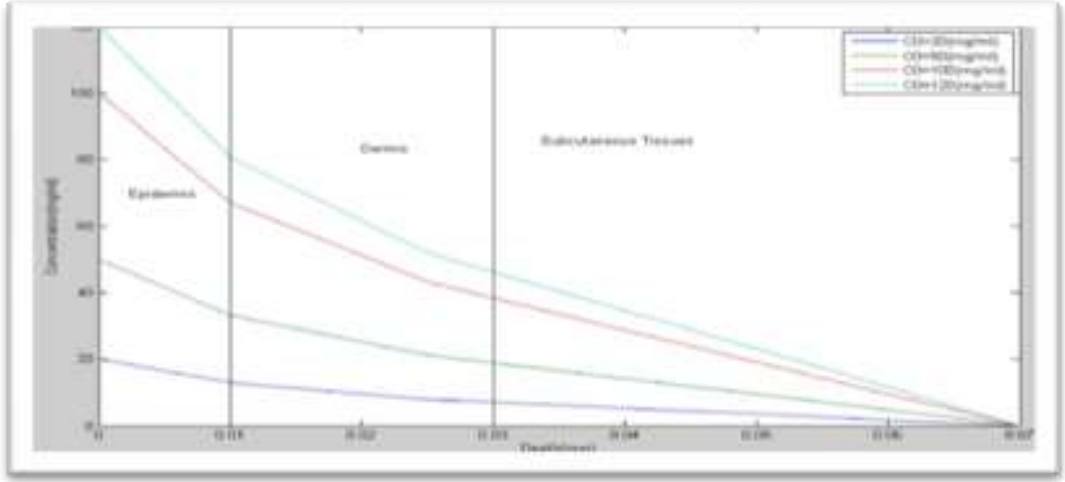

Figure [3]

Steady state drug concentration distribution in skin layer. The result for the linear diffusion with $T_{1}(C)=0$ $\mathrm{gm} / \mathrm{mm}^{3} \mathrm{sec}, T_{2}(\mathrm{C})=2 * 10^{-3} \mathrm{gm} / \mathrm{mm}^{3} \mathrm{sec}, \mathrm{T}_{3}(\mathrm{C})=3^{*} 10^{-3} \mathrm{gm} / \mathrm{mm}^{3} \mathrm{sec}, B_{1}=0 \mathrm{gm} / \mathrm{mm}^{3} \mathrm{sec}, B_{2}=1^{*} 10^{-2} \mathrm{gm} / \mathrm{mm}^{3} \mathrm{sec}$, $\mathrm{B}_{3}=2 * 10^{-2} \mathrm{gm} / \mathrm{mm}^{3} \mathrm{sec}, \mathrm{D}_{1}=1.83 * 10^{-4} \mathrm{~mm}^{2} / \mathrm{sec}, \mathrm{D}_{2}=4.16^{*} 10^{-4} \mathrm{~mm}^{2} / \mathrm{sec}, \mathrm{D}_{3}=6.04 * 10^{-4} \mathrm{~mm}^{2} / \mathrm{sec}$.

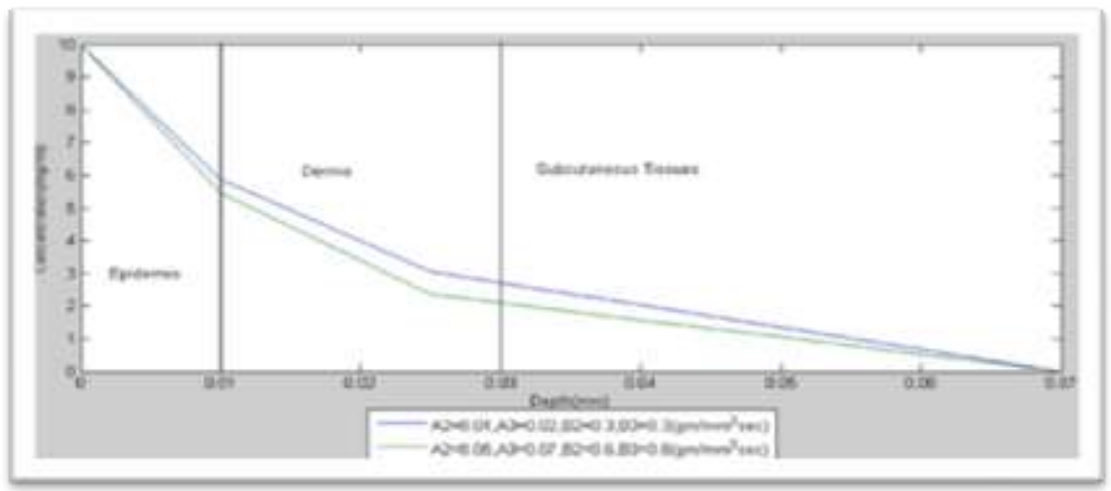

Figure [4]

Concentration Profile for initial concentration of $10(\mathrm{mg} / \mathrm{ml})$ with influence of absorption coefficients on drug concentration in the skin. The result for Linear diffusion with $D_{1}=1.83 * 10^{-4} \mathrm{~mm}^{2} / \mathrm{sec}, D_{2}=4.16^{*} 10^{-4} \mathrm{~mm}^{2} / \mathrm{sec}$, $\mathrm{D}_{3}=6.04 * 10^{-4} \mathrm{~mm}^{2} / \mathrm{sec}$.

The values of physical and physiological parameters (shown in table 1 and 2 )[18] are feasible and fall within the admissible ranges and used for finding concentration profile in the dermal region.Graphs have been plotted using MATLAB software between field concentration $\mathrm{C}^{(i)}(\mathrm{i}=1,2,3)$ and thickness of skinx. Figure (2) and (3) show the field concentration with respect to depth $\mathrm{x}$.The steepness of curve in these figures decreases as we 
move from outer surface to inner boundary, which is due to the effect of inner boundary condition where concentration decreases with the increase in distance from the outer boundary. The effect of absorption coefficient and diffusivity can also be observed from these figures increase in absorption decreases the concentration. Also increase in diffusion increases the concentration as shown in figure (4).

The slope of these curve decreases at the interfaces, this effect is due to the change in properties of each sub-region. This approach is intended to help researchers develop highly effective drug formulations and more accurate dosing regimens.

Table 1: Set of different values of diffusivity in each layer.

\begin{tabular}{|c|c|c|}
\hline$D_{1}\left(\mathrm{~mm}^{2} \mathrm{sec}^{-1}\right)$ & $D_{2}\left(\mathrm{~mm}^{2} \mathrm{sec}^{-1}\right)$ & $D_{3}\left(\mathrm{~mm}^{2} \mathrm{sec}^{-1}\right)$ \\
\hline $1.83^{* 10^{-4}}$ & $4.16^{*} 10^{-4}$ & $6.04 * 10^{-4}$ \\
\hline $5.83 * 10^{-4}$ & $7.16^{*} 10^{-4}$ & $9.04 * 10^{-4}$ \\
\hline $4.0 * 10^{-3}$ & $7.05 * 10^{-3}$ & $9.05 * 10^{-3}$ \\
\hline
\end{tabular}

Table 2: Different values of $T_{i}(C)$ and $B_{i}$ in each layer.

\begin{tabular}{|c|c|}
\hline $\begin{array}{c}\text { Tissue absorption rate of drug T(C) } \\
\left(\mathbf{g m} * \mathbf{m m}^{-3} \mathbf{s e c}^{-1}\right)\end{array}$ & $\begin{array}{c}\text { Drug Intake rate by blood(B) } \\
\left(\mathbf{g m} * \mathbf{m m}^{-3} \mathbf{s e c}^{-1}\right)\end{array}$ \\
\hline $\mathbf{T}_{\mathbf{2}}(\mathbf{C})=\mathbf{1 . 0} * \mathbf{1 0}^{-4}$ & $\mathbf{B}_{2}=\mathbf{2 . 0} * \mathbf{1 0}^{-3}$ \\
\hline $\mathbf{T}_{3}(\mathbf{C})=\mathbf{4 . 0} * \mathbf{1 0}^{-4}$ & $\mathbf{B}_{3}=\mathbf{5 . 0} * \mathbf{1 0}^{-3}$ \\
\hline $\mathbf{T}_{2}(\mathbf{C})=\mathbf{5 . 0} * \mathbf{1 0}^{-4}$ & $\mathbf{B}_{2}=\mathbf{4 . 0} * 10^{-3}$ \\
\hline $\mathbf{T}_{3}(\mathbf{C})=\mathbf{8 . 0} * \mathbf{1 0}^{-4}$ & $\mathbf{B}_{3}=\mathbf{7 . 0} * \mathbf{1 0}^{-3}$ \\
\hline $\mathbf{T}_{2}(\mathbf{C})=\mathbf{2 . 0} * \mathbf{1 0}^{-3}$ & $\mathbf{B}_{2}=\mathbf{1 . 0} * \mathbf{1 0}^{-2}$ \\
\hline $\mathbf{T}_{3}(\mathbf{C})=\mathbf{5 . 0} * \mathbf{1 0}^{-3}$ & $\mathbf{B}_{2}=\mathbf{2 . 0} * \mathbf{1 0}^{-2}$ \\
\hline
\end{tabular}

\section{Appendix A:}

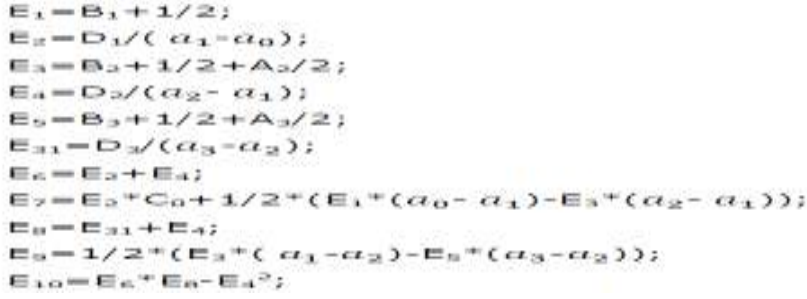

\section{References:}

[1] Agrawal G. , Development, fabrication and evaluation of TDDS- A review,2009 (vol.7,Issue 5).

[2] Chao K.N., Eisley J.G. \& Yang W.J.(1973) Heat and water migration in regional skin and subcutaneous tissues , Bio-MechSypm ASME,69-72.

[3] Chao K.N., Yang W.J.(1975) Response of skin and tissue temperature in sauna and stem bath, Bio-MechSypm ASME, 69-71.

[4] Cleary GW, Lange RS, Wise DL. Medical application of controlled release, CRC Press, Boca Raton, Florida.1984, Vol I: 203-45

[5] Gurang D.B., Saxena V.P. and Adhikary P.R. (2009) FEM approach to one dimensional unsteady state temperature distribution in human dermal parts with quadratic shape functions. J App Math and Info, 27,301-313

[6] Guy and Hadgraft, Transdermal Drug Delivery Development, Issue and research initiatives.

[7] J. LEE. J.R. KING., T.G. ROGERS. 1996. A multiple-pathway model for the diffusion of drugs in skin. IMA J. Math. Applied in Med. \& Bio. 13, 127-150.

[8] Kalia , Y.N. \& Guy , R.H. (2001) Modeling Transdermal drug release , Adv. Drug Delivery Rev, 48, $159-172$.

[9] Missle , P.J.(2000) Finite element modeling of diffusion \& partitioning in biological system : The infinite composite medium problem , Ann BomedEng, 48,1307-1317.

[10] Myres G.E.(1971) Analytical methods in conduction heat transfer, Mc-Graw Hill Co,New York pp 320-428.

[11] Patani, G.A. and Chien, Y.W., In; Swerbrick, J. and Boylon, J.C., Eds., Encyclopedia of Pharmaceutical Technology, Vol. 18, Marcel Dekker Inc., New York, 1999, 317-320, 329

[12] Pathan I Bashir, Setty C Mallikarjuna. Chemical Penetration Enhancers for Transdermal Drug Delivery Systems .Trop J Pharm Res. 2009, 8 (2): 173-179.

[13] Patrick J.Sinko, Martin's Physical Pharmacy and Pharmaceutical Sciences ( $5^{\text {th }}$ Edition).

[14] Saxena V.P. \&Bindra , J.S. (1987) Quadratic shape function in variational finite element approach to heat distribution incutaneous and subcutaneous tissues. Ind J Pure and ApplMaths, 18,846-855.

[15] Saxena V.P. \&Arya, D., Steady - State Head Distribution in epidermis, dermis \& Sub dermal Tissues Journal Theor. Biol. Vol $89[1981] 423-432$.

[16] Saxena V.P. , Temperature Distribution in Human Skin \& Sub dermal Tissue Journal Theory Biol. Vol. 102[1983]277-286.

[17] Saxena V.P. \& Sharma (2011), one dimensional drug distribution in human dermal region.

[18] Y GLV, Liu J, Gao Y H and Xu 13 (2006), Modeling of Transdermal Drug Delivery with a Micro-needle array, J.Micromech , Microeng 16,2492-2501. 